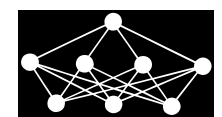

\title{
TRANSITION MODES BETWEEN SPIKING AND BURSTING IN A PACEMAKER NEURON
}

\author{
W. Ye, S. Liu, X. Liu
}

\begin{abstract}
Central pattern generators (CPGs) play an important role in controlling rhythmic movements in vivo. Increased insight into mechanisms of CPGs can be obtained by perturbing neuron activities so as to study a range of behaviors. By applying this method, a series of simulations were performed to research different transition modes between firing patterns in a pacemaker neuron model of stomatogastric ganglion (STG). Firstly, with the perturbation of parameters in model, such as external stimulus, parameters in compartments and connection between compartments, different firing patterns and bifurcation of inter-spike intervals (ISIs) were obtained to exhibit the impact of single parameter on the transions between spiking and bursting. Moreover, perturbing two parameters $g_{\mathrm{Ca}}$ and $I_{\text {ext }}$ simultaneously induced the continuous variation of the bifurcation mode, which implied the crucial role of calcium channel in regulating the rhythm generation. Finally, a two-dimensional parameter space $\left(g_{\mathrm{Ca}}, I_{\text {ext }}\right)$ was constructed by spike-counting method to capture the distribution of the firing patterns and different transition mode between them in a comprehensive aspect. In this parameter space, three basic transition modes were concluded: bifurcation ring, period-doubling mode and period-adding mode.
\end{abstract}

Key words: central pattern generators, stomatogastric ganglion, inter-spike intervals, period-doubling bifurcation

Received: March 25, 2014

DOI: $10.14311 / \mathrm{NNW} .2017 .27 .012$

Revised and accepted: May 2, 2017

\section{Introduction}

The stomatogastric ganglion (STG) that lies at the dorsal surface of the foregut in crustaceans is comprised of two central pattern generators (CPGs): pyloric and gastric mill CPGs. These CPGs are believed to play a part in controlling some rhythmic and repetitive movements, such as locomotion and feeding $[15,19]$. In general, endogenous oscillator CPGs are driven by pacemaker neurons that fire rhythmic spike bursts [21]. Therefore, it is crucial to acquaint the firing patterns of pacemaker neurons and transition patterns between them, for understanding how CPGs regulate different motor patterns.

*Weijie Ye; Shenquan Liu - Corresponding author; Xuanliang Liu; School of Mathematics, South China University of Technology, Guangzhou, China, E-mail: mashqliu@scut.edu.cn 
The mechanisms behind how firing patterns vary are of interest because the firing pattern is likely to encode information which is transmitted to target neurons and these kinds of variation maybe induce different behaviors or different states of movement. Nevertheless, due to the difficulty of experimental research in neuroscience, these mechanisms are far away clear. As a result, much attention is focused on computational method. Plenty of models, which contributed to understanding the effect of pacemaker neurons, have been constructed and researched, such as the R15 neuron in Aplysia [1,23] and inspiratory pacemaker neuron in pre-Botzinger [6]. Canavier and her colleagues [7] even demonstrated a perioddoubling transition mode between spiking and bursting in R15 neuron. Besides single neuron model, a great many complicated simulations were achieved by constructing network models for understanding the contribution of pacemaker neurons in network $[2,5,11,18,19,22]$. Network models constructed by Weaver et al. [22] and Soto-Trevino et al. [18] provided a comprehensive way in studying the effect of firing patterns of pacemaker kernels on other neurons. Recently, the research about pacemaker neurons went deep into the impact of external environmental conditions such as temperature [17]. Moreover, some researchers like Bedrov et al. [4] and Falcke et al. [8] focused their attention on dynamical properties such as limit cycle, attractor, providing mathematical insight into the firing modes. Although lots of experiments and models have been completed, the mechanisms behind variation of firing patterns remain less obvious.

In this work, we simulated a compartmental pacemaker neuron model introduced by Maran et al. [13], and studied the transition modes between different firing patterns. The index of inter-spike intervals (ISIs) was utilized to describe the variation of firing patterns $[9,14,16]$. In addition, we used the spike-counting method to construct a two-dimensional parameter plane which can provide a comprehensive way of understanding the distribution of firing patterns and the transition modes between them $[3,20]$.

\section{Model descriptions}

The pyloric pacemaker neuron model simulated here is the same as the model introduced by Maran et al. [13]. It includes four compartments: soma, primary neurite, dendrite and axon, and its morphology is shown in Fig. 1. Each compartment has its corresponding ion channels. The soma only has a leakage channel (Leak), while the primary neurite has leakage channel and a very slow potassium channel. There are five kinds of channel in the dendrite: calcium channel (Ca), leakage channel (Leak), calcium-dependent potassium channel (KCa), fast potassium channel (Kf) and transient outward potassium channel (A). The axon includes the traditional Hodgkin-Huxley sodium and delayed-rectifier potassium channels ( $\mathrm{Na}$ and $\mathrm{Kdr}$ ) as well as leakage channel (Leak). The equations and parameters of model are given in Appendix.

All simulations were implemented in Python 2.7 using a fourth order RungeKutta method with integration time steps of $0.01 \mathrm{~ms}$. For the sake of accuracy, the first $20000 \mathrm{~ms}$ of each firing was discarded. 


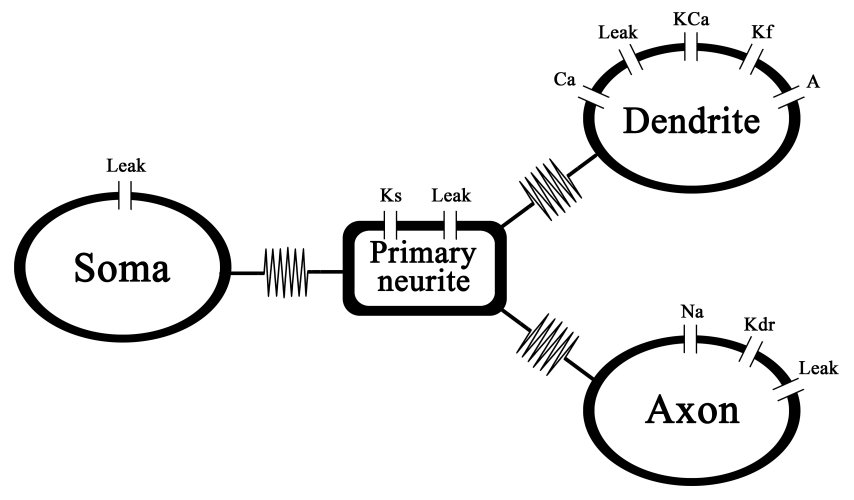

Fig. 1 The structure and ion channels of the four compartments neuron model in stomatogastric ganglion

\section{Results}

\subsection{Influence of external stimulus on compartmental model}

Different current injection could vary the activity of neurons from resting to bursting [18]. For understanding the effect of external stimulus, we performed some simulations by injecing different external stimulus. Insets in Fig. 2a show several
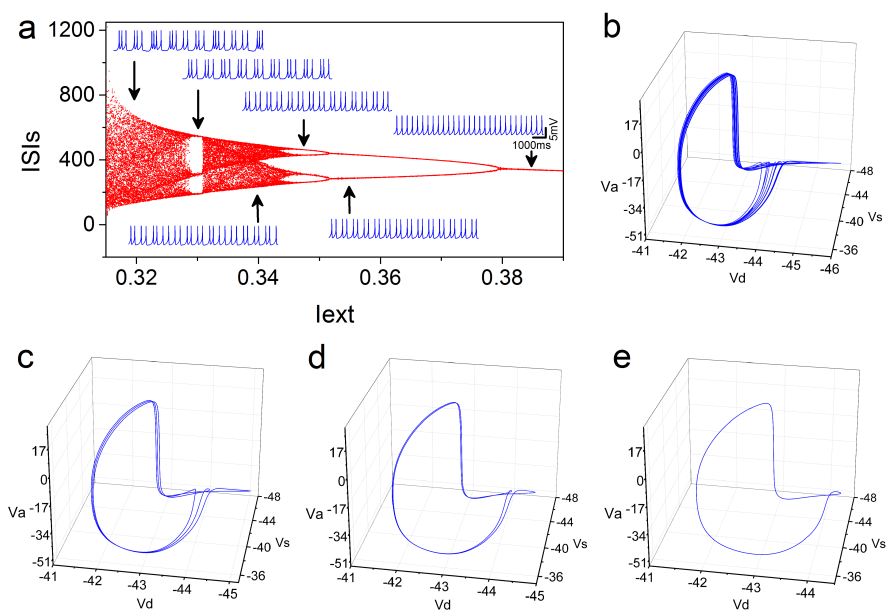

Fig. 2 The effect of external stimulus on the transition between different firing patterns. (a) Bifurcation diagrams of ISIs influenced by $I_{\mathrm{ext}}$. Insets illustrate firing patterns in different $I_{\mathrm{ext}}: 0.32,0.3307,0.34,0.347,0.355$ and $0.385 \mathrm{nA}$, respectively. (b) $3 D$ phase portrait of chaotic motion when $I_{\mathrm{ext}}=0.32 n \mathrm{~A}$. (c)-(e) $3 D$ phase portraits of periodic motion with different periods when $I_{\mathrm{ext}}=0.347 \mathrm{nA}$ (c), $0.355 \mathrm{nA}(d)$ and $0.385 n \mathrm{~A}(\mathrm{e})$. 
firing patterns of neuron model after applying distinct strengths of external stimulus. When $I_{\text {ext }}$ is $0.32 \mathrm{nA}$, the model exhibits chaotic bursting pattern. All bursts in this series seem different, and the number of spikes in a burst could be 2,3 or 4 . At a higher strength of $I_{\text {ext }}$ input, i.e. $I_{\text {ext }}=0.33 \mathrm{nA}$, a regular bursting pattern is observed, while the firing pattern turns into tonic-spiking as $I_{\text {ext }}$ exceeds $0.385 \mathrm{nA}$. These firing patterns all correspond to different regions of bifurcation diagram that plot ISIs versus $I_{\text {ext }}$ (Fig. 2a). With $I_{\text {ext }}$ increasing, an inverse period-doubling cascade is observed with the chaotic regions and periodic motions alternately appearing. In the parameter range $0.316<I_{\text {ext }}<0.347$, the chaotic firing patterns are the main pattern (Fig. 2b). When $I_{\text {ext }}>0.347 \mathrm{nA}$, the model exhibits periodic motions (Fig. 2c-e).

\subsection{Contribution of parameters in neuron to transition mode of firing patterns}

\subsubsection{The impact of parameters in compartments}

Having established the effects of varying $I_{\text {ext }}$ on the firing patterns, we proceeded to examine the effects of some parameters in compartments. We found that many parameters in compartments have influence on the firing patterns of neuron. Fig. 3 shows six bifurcation diagrams which plot ISIs as the function of some parameters. It is clear that the variation of some conductance and equilibrium potentials make
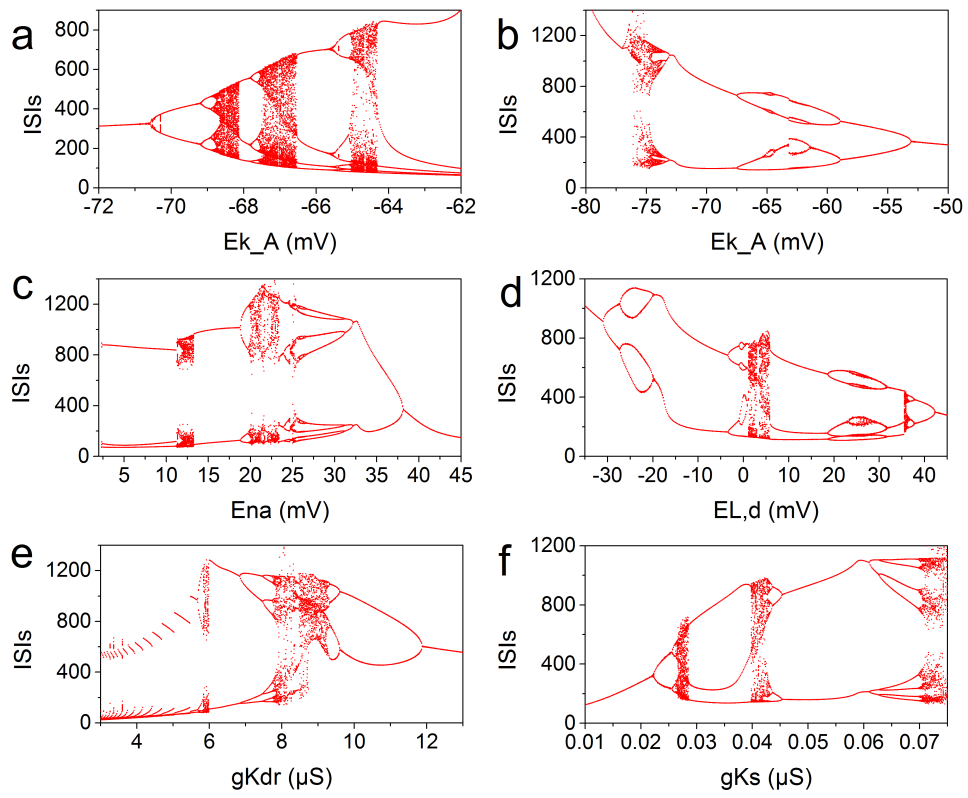

Fig. 3 Bifurcation diagrams of ISIs obtained by perturbing various parameters in compartments: (a) $E_{\mathrm{K}_{-} \mathrm{Kdr}}$, (b) $E_{\mathrm{K}_{-} \mathrm{A}}$, (c) $E_{\mathrm{Na}}$, (d) $E_{\mathrm{L}, \mathrm{d}}$, (e) $g_{\mathrm{Kdr}}$, (f) $g_{\mathrm{Ks}}$. 
pacemaker neuron exhibit rich dynamic behavior. Fig. 3a shows a typical bifurcation diagram that performs continuous period-doubling bifurcation as $E_{\mathrm{K} \_K d r}$ increases. In contrast, some interesting "ring-shaped bifurcations", which firstly exhibit period-doubling bifurcation and then induce inverse period-doubling motion, have occurred in other diagrams, such as the ranges $-68.2<E_{\mathrm{K}_{-} \mathrm{A}}<-57.5$, $-27.5<E_{\mathrm{L}, \mathrm{d}}<-20$ and $6.8<g_{\mathrm{Kdr}}<11.6$ (Fig. 3b-Fig. 3f). We refer to it as a "bifurcation ring". In bifurcation rings, there also exists some dynamic behaviors. For instance, the nested bifurcation rings in the parameter range $-65<E_{\mathrm{K}_{-} \mathrm{A}}<-62$, and chaotic motion and periodic motion in the ranges $7.5<g_{\mathrm{Kdr}}<9.6,0.25<g_{\mathrm{Ks}}<0.43$. These dynamic behaviors reveal abundant transition modes of firing patterns when parameters are perturbed.

\subsubsection{The effect of connections between compartments}

The coupling strength is an important parameter in transferring excitement between compartments. For understanding how coupling strength affects the firing patterns, three numerical simulations were done. As we can see in Fig. 4, three bifurcation diagrams are produced as a result of perturbing coupling conductances
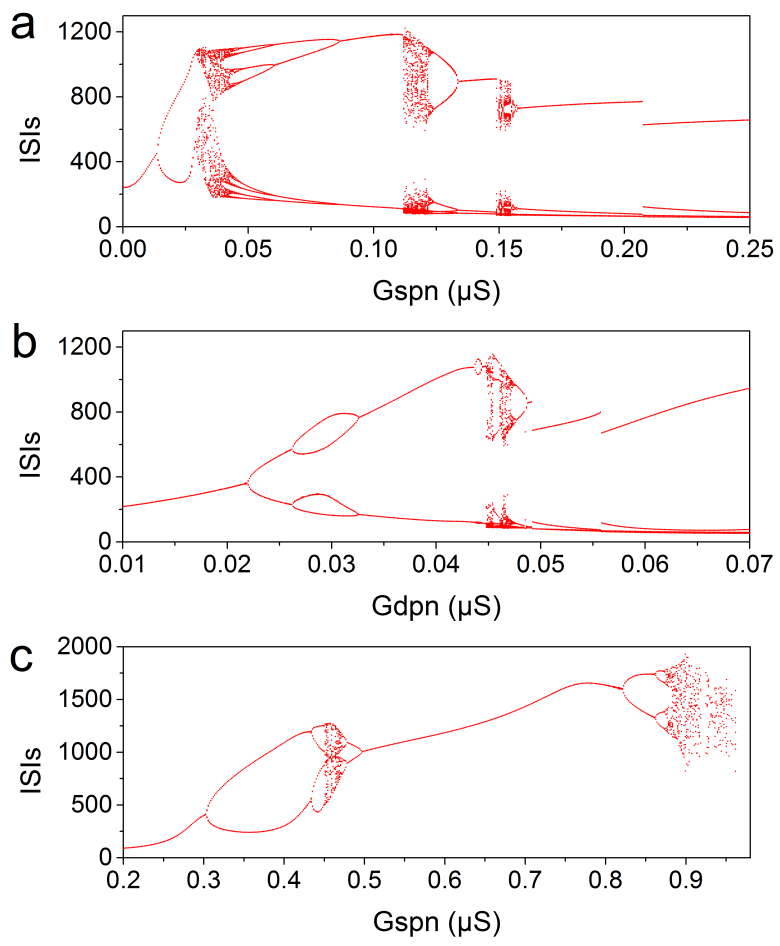

Fig. 4 The ISIs bifurcation diagrams with the change of the coupling conductances: (a) $G_{\mathrm{s}, \mathrm{pn}}$, (b) $G_{\mathrm{d}, \mathrm{pn}}$ and (c) $G_{\mathrm{a}, \mathrm{pn}}$. 
$G_{\mathrm{s}, \mathrm{pn}}, G_{\mathrm{d}, \mathrm{pn}}$ and $G_{\mathrm{a}, \mathrm{pn}}$. No matter which connection it is, it can be observed that weak coupling just induces tonic-spiking firing. As coupling conductance increased, the model exhibits period-doubling motions. In the parameter ranges $0.012<G_{\mathrm{s}, \mathrm{pn}}<0.11,0.026<G_{\mathrm{d} \text {,pn }}<0.031$ and $0.305<G_{\mathrm{a}, \mathrm{pn}}<0.5$, bifurcation rings can be observed. The transition mode of firing patterns induced by $G_{\mathrm{a}, \mathrm{pn}}$ is different from the others, for both relatively weak coupling and strong coupling could generate rich dynamic behavior (Fig. 4c). In relative weak coupling of $G_{\mathrm{a}, \mathrm{pn}}$, there are two nested chaotic rings in the bifurcation ring. When $G_{\mathrm{a}, \mathrm{pn}}>0.5$, the model ends the bifurcation ring and generates period-one motion. The most interesting thing is, strong coupling makes the model produce period-doubling cascade again. Finally, it turns into chaotic motion.

\subsection{The calcium conductance $g_{\text {Ca }}$ induced continuous change of the bifurcation}

A set of bifurcation diagrams were constructed by plotting ISIs versus $I_{\text {ext }}$ with different $g_{\mathrm{Ca}}$ for detecting impact of $g_{\mathrm{Ca}}$ on bifurcation. The results of simulation are shown in Fig. 5, from which we can see that bifurcation diagrams have a continuous change by increasing $g_{\mathrm{Ca}}$. When $g_{\mathrm{Ca}}$ is $0.0198 \mu \mathrm{S}$, the model possesses two firing patterns: single spike firing and burst firing that have ISIs of two periods (Fig. 5a). In this chart, ISIs bifurcate into two periods, then gathered together and forms a bifurcation ring. While $g_{\mathrm{Ca}}$ increases, the bifurcation ring enlarges and produced new nested bifurcation rings, indicating that new firing patterns occur (Fig. 5b-Fig. 5d). As $g_{\mathrm{Ca}}=0.0240 \mu \mathrm{S}$, bifurcation rings in diagram break up and shape a new bifurcation mode (Fig. 5e). Three new bifurcation rings come up in the parameter ranges $0.305<I_{\text {ext }}<0.36$ and $0.545<I_{\text {ext }}<0.604$, when $g_{\text {Ca }}=$ $0.02525 \mu \mathrm{S}$ (Fig. $5 \mathrm{~g}$ ). As $g_{\mathrm{Ca}}$ increases from $0.02550 \mu \mathrm{S}$ to $0.02715 \mu \mathrm{S}$ (Fig. $5 \mathrm{~h}-$ Fig. 5o), the bifurcation rings gradually enlarge again and finally crash. Whenever bifurcation rings break up in this procedure, a chaotic motion would always be evoked at the position that bifurcation rings crash (Fig. 5e, Fig. 5k, Fig. 5m).

\subsection{Two-dimensional parameter space analysis}

From the aforementioned results, we found that the STG pacemaker neuron can exhibited rich dynamic behaviors by changing different parameters. However, it is not easy to obtain a comprehensive understanding of the transition between distinct firing patterns if we only focus on the bifurcation modes produced by single parameter. In this section, we present a two-dimensional parameter space diagram which is plotted on a $500 \times 500$ grid of parameter $\left(g_{\mathrm{Ca}}, I_{\text {ext }}\right)$ by spikecounting method (Fig. 6). This means that $25 \times 10^{4}$ simulations have been done for constructing it. This plane can also explain the continued change of the bifurcation diagrams in Fig. 5.

It is obvious that the parameter plane is partitioned into different regions, corresponding to different behaviors. In general, the diagram illustrates four kinds of firing patterns: quiescence (0), tonic-spiking (1), bursting (2-34) and chaotic firing (35). We conclude the transition modes of these activities into three basic patterns. 
Ye W., Liu S., Liu X.: Transition modes between spiking and bursting...
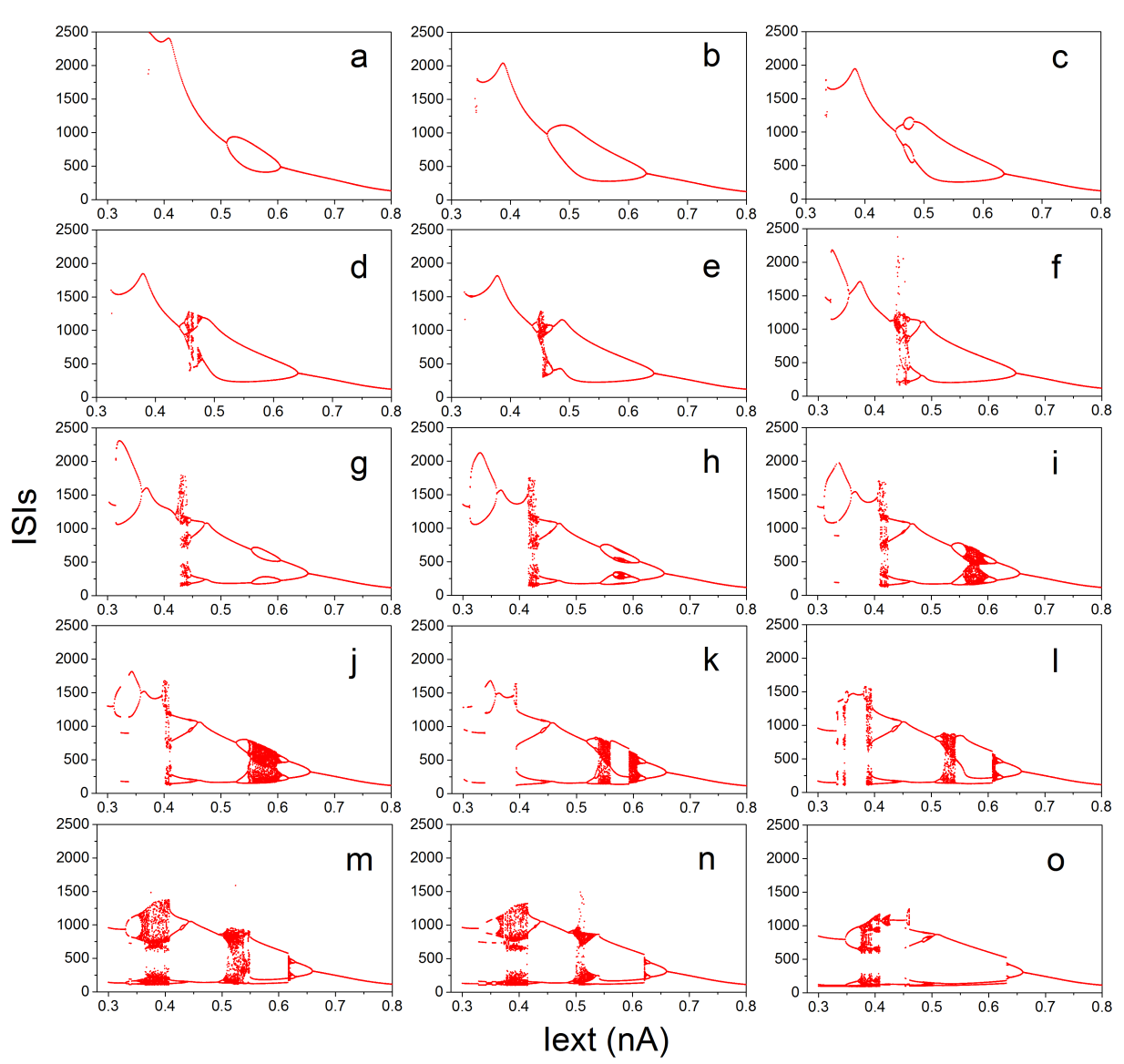

Fig. 5 ISIs bifurcation diagrams that plotted ISIs versus Iext at different value of calcium channel conductance $g_{\mathrm{Ca}}$. The value of $g_{\mathrm{Ca}}$ from up to down and from left to right are $0.0198(a), 0.02260(b), 0.02320(c), 0.02380(d), 0.02400(e), 0.02460$ $(f), 0.02525(\mathrm{~g}), 0.02550(\mathrm{~h}), 0.02565(\mathrm{i}), 0.02585(\mathrm{j}), 0.02605(\mathrm{k}), 0.02630(\mathrm{l})$, $0.02655(\mathrm{~m}), 0.02670(\mathrm{n}), 0.02715(\mathrm{o})$.

i) Bifurcation ring. In subregion A of Fig. 6, periods in vertical direction are organized as $1 \rightarrow 2 \rightarrow 1$. Similarly, in the middle of the parameter-plane, periods change by a sequence $1 \rightarrow 2 \rightarrow 4 \rightarrow 2 \rightarrow 1$. This transition mode combine a period-doubling bifurcation and an inverse period-doubling motion, forming a ring-shaped structure.

ii) Period-doubling model. As we can see in subregion B, a more complicated structure can be observed. Now we move from left to right, the variation of firing patterns are organized by the sequence: $2 \rightarrow 4 \rightarrow$ chaos $\rightarrow 3 \rightarrow 6 \rightarrow$ chaos $\rightarrow 4$. We can conclude this transition mode into a universal version: $\cdots \rightarrow n \rightarrow 2 n \rightarrow$ chaos $\rightarrow n+1 \rightarrow 2(n+1) \rightarrow$ chaos $\rightarrow n+2 \rightarrow \cdots$. 
iii) Period-adding mode. The feature of this transition mode is that periods increase by a factor of $\mathrm{i}$, and organize as $\cdots \rightarrow n \rightarrow n+i \rightarrow n+2 i \rightarrow$ $n+3 i \rightarrow \cdots$. Taking the subregion $C$ as a example, two period-adding modes are observed. The period of the top are change by a sequence $4 \rightarrow 4+2 \rightarrow$ $4+4 \rightarrow 4+6$, while the bottom one are organized as $2 \rightarrow 2+1 \rightarrow 2+2 \rightarrow 2+3$.

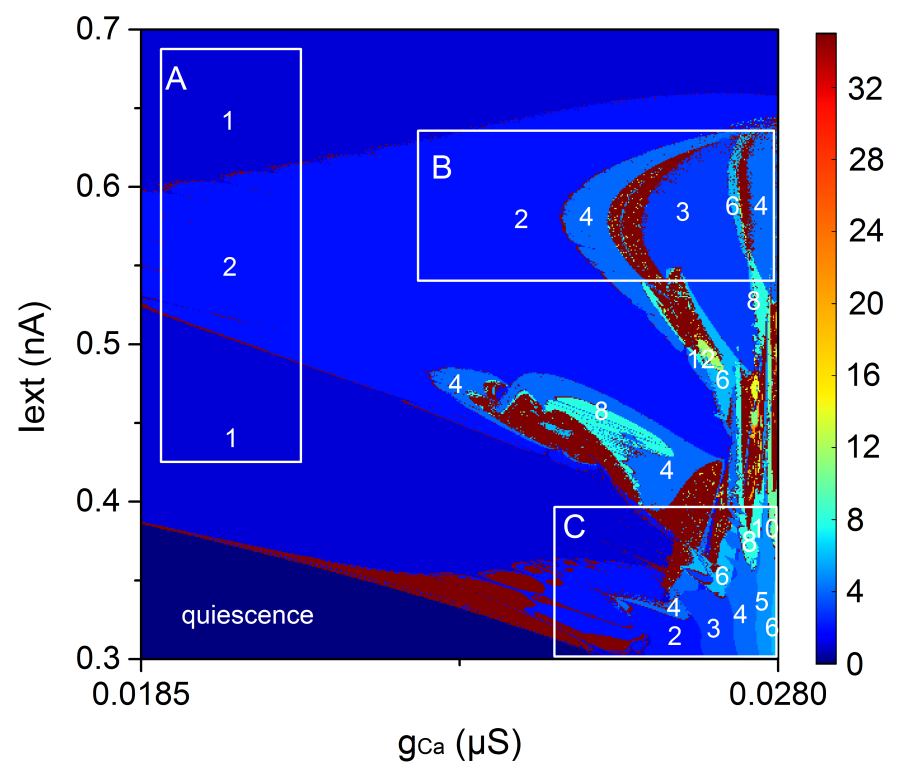

Fig. $6\left(g_{\mathrm{Ca}}, I_{\mathrm{ext}}\right)$ parameter-space. The color bar on the right presents the spikenumber. 0 represents quiescence, 1 stands for tonic-spiking patterns, and 2-34 is the bursting pattern, while 35 represents chaotic firing.

\section{Discussion}

Based on the STG pacemaker neuron model, a plethora of firing patterns and the transition modes of the model were investigated in this study. The results provide insight into how CPGs control rhythmic movements by varying firing behaviors. The factors affecting the firing patterns include external stimulus and parameters in the neuron model. Both external stimulus and parameters in neuron could induce the bifurcation phenomenon of ISIs, in spite of none of these phenomena is the same. It seems that bifurcation ring is a typical transition mode of firing patterns in pacemaker neuron. The bifurcation ring implies a mechanism that the excitability of neuron suddenly increase and then return to oringinal state with some parameters varying. This mechanism may be related to some movements in STG.

By use of spike-counting method, a two-dimensional parameter space diagram was constructed to illustrate the variation of firing patterns when two parameters 
Ye W., Liu S., Liu X.: Transition modes between spiking and bursting...

were varied. This diagram also explains the continuous change of the bifurcation diagram in Fig. 5. As a result, three basic transition modes were concluded: bifurcation ring, period-doubling mode and period-adding mode. We can find that the bifurcation phenomena in Fig. 2-Fig. 5 all are the combination of these three modes. However, it is far away clear that how the parameters vary in vivo. In recent, some researches have reported that animals can homeostatically control the parameters so as to maintain a balance state $[10,12]$. Thus, the transition patterns found in this study may just disclose a corner of the total impacts of parameters. When covarying more parameters, there may be some other new transitions patterns can be found. Further research should consider this limitation and find new transition modes in higher dimensional parameter space.

\section{Acknowledgement}

This work was supported by the National Natural Science Foundation of China (Grant No. 11172103).

\section{References}

[1] ADAMS W.B. Slow depolarizing and hyperpolarizing currents which mediate bursting in aplysia neurone R15. J Physiol, 1985, 360, pp. 51-68, doi: 10.1113/jphysiol.1985. sp015603.

[2] AYALI A., HARRIS-WARRICK R.M. Monoamine control of the pacemaker kernel and cycle frequency in the lobster pyloric network. J Neurosci, 1999, 19(15), pp. 6712-6722, 1999. Available from: http://www.jneurosci.org/content/19/15/6712.full.pdf

[3] BARRIO R., SHILNIKOV A. Parameter-sweeping techniques for temporal dynamics of neuronal systems: case study of hindmarsh-rose model. J Comput Neurosci, 2011, 1(1), pp. 1-22, doi: $10.1186 / 2190-8567-1-6$.

[4] BEDROV Y. A., DICK O. E., NOZDRACHEV A.D., AKOEV G.N. Method for constructing the boundary of the bursting oscillations region in the neuron model. Biol Cybern, 2000, 82(6), pp. 493-507, doi: 10.1007/s004220050602.

[5] BEM T., LE-FEUVRE Y., SIMMERS J., MEYRAND P. Electrical coupling can prevent expression of adult-like properties in an embryonic neural circuit. J Neurophysiol, 2002, 87(1), pp. 538-547. Available from: http://jn.physiology.org/content/87/1/538.full. pdf

[6] BUTERA R.J., RINZEL J., SMITH J.C. Models of respiratory rhythm generation in the prebotzinger complex. I. bursting pacemaker neurons. J Neurophysiol, 1999, 82(1), pp. 382-397. Available from: http://jn.physiology.org/content/jn/82/1/382.full.pdf

[7] CANAVIER C.C., CLARK J.W., BYRNE J.H. Routes to chaos in a model of a bursting neuron. Biophys J, 1991, 57(6), pp. 1245-1251, doi: 10.1016/S0006-3495(90)82643-6.

[8] FALCKE M., HUERTA R., RABINOVICH M.I., ABARBANEL H.D., ELSON R.C., SELVERSTON A.I. Modeling observed chaotic oscillations in bursting neurons: the role of calcium dynamics and IP3. Biol Cybern, 2000, 82(6), pp. 517-527, doi: 10.1007/ s004220050604.

[9] FEUdel U., NEIMAN A., PEI X., WOJTENEK X., BRAUN H., HUBER M., MOSS F. Homoclinic bifurcation in a hodgkin-huxley model of thermally sensitive neurons. Chaos, 2000, 10(1), pp. 231-239, doi: 10.1063/1.166488.

[10] HAIDER B., DUQUE A., HASENSTAUB A.R., MCCORMICK D.A. Neocortical network activity in vivo is generated through a dynamic balance of excitation and inhibition. $\mathrm{J}$ Neurosci, 2006, 26(17), pp. 4535-4545, doi: 10.1523/JNEUROSCI.5297-05.2006. 


\section{Neural Network World 2/2017, 247-258}

[11] KEPLER T.B., MARDER E., ABBOTT L.F. The effect of electrical coupling on the frequency of model neuronal oscillators. Science, 1990, 248(4951), pp. 83-95, doi: 10.1126/ science. 2321028 .

[12] LAMB D.G., CALABRESE R.L. Correlated conductance parameters in leech heart motor neurons contribute to motor pattern formation. PloS One, 2013, 8(11), pp. e79267, doi: 10. 1371/journal.pone.0079267.

[13] MARAN S.K., SIELING F.H., DEMLA K., PRINZ A.A., CANAVIER C.C. Responses of a bursting pacemaker to excitation reveal spatial segregation between bursting and spiking mechanisms. J Comput Neurosci, 2011, 31(2), pp. 419-440, doi: 10.1007/ s10827-011-0319-y.

[14] NEIMAN A.B., DIERKES K., LINDNER B., HAN L., SHILNIKOV A.L. Spontaneous voltage oscillations and response dynamics of a hodgkin-huxley type model of sensory hair cells. J Math Neurosci, 2011, 1(11), p. 11, doi: 10.1186/2190-8567-1-11.

[15] OPRISAN S.A., THIRUMALAI V., CANAVIER C.C. Dynamics from a time series: Can we extract the phase resetting curve from a time series? Biophys J, 2003, 84(5), pp. 2919-2928, doi: 10.1016/S0006-3495(03) 70019-8.

[16] POSTNOVA S., VOIGT K., BRAUN H.A. Neural synchronization at tonic-to-bursting transitions. J Biol Phys, 2007, 33(2), pp. 129-143, doi: 10.1007/s10867-007-9048-x.

[17] RINBERG A., TAYLOR A.L., MARDER E. The effects of temperature on the stability of a neuronal oscillator. PLoS Comput Biol, 2013, 9(1), pp. e1002857, doi: 10.1371/journal. pcbi. 1002857.

[18] SOTO-TREVIÑO C., RABBAH P., MARDER E., NADIM F. Computational model of electrically coupled, intrinsically distinct pacemaker neurons. J Neurophysiol, 2005, 94(1), pp. 590-604, doi: 10.1152/jn.00013.2005.

[19] SOTO-TREviÑo C., THOROUGHMAN K.A., MARDER E., ABBOTT L.F. Activitydependent modification of inhibitory synapses in models of rhythmic neural networks. Nat Neurosci, 2001, 4(3), pp. 297-303, doi: 10.1038/85147.

[20] STORACE M., LINARO D., DE LANGE E. The Hindmarsh-Rose neuron model: bifurcation analysis and piecewise-linear approximations. Chaos, 2008, 18(3), pp. 033128, doi: 10.1063/ 1.2975967.

[21] WEAVER A.L., HOOPER S.L. Follower neurons in lobster (panulirus interruptus) pyloric network regulate pacemaker period in complementary ways. J Neurophysiol, 2003, 89(3), pp. 1327-1338, doi: 10.1152/jn.00704.2002.

[22] WEAVER A.L., HOOPER S.L.Relating network synaptic connectivity and network activity in the lobster (panulirus interruptus) pyloric network. J Neurophysiol, 2003, 90(4), pp. 23782386, doi: 10.1152/jn.00705.2002.

[23] YU X., BYRNE J.H., BAXTER D.A. Modeling interactions between electrical activity and second-messenger cascades in aplysia neuron r15. J Neurophysiol, 2004, 91(5), pp. 22972311, doi: 10.1152/jn.00787.2003.

\section{A. Neuron model in detail}

The compartmental model is described by the following Hodgkin-Huxley type equations:

$$
\begin{aligned}
& C_{\mathrm{m}} \frac{\mathrm{d} V_{\mathrm{s}}}{\mathrm{d} t}=-\left(I_{\mathrm{L}, \mathrm{s}}+I_{\mathrm{s}, \mathrm{pn}}\right), \\
& C_{\mathrm{m}} \frac{\mathrm{d} V_{\mathrm{pn}}}{\mathrm{d} t}=-\left(I_{\mathrm{L}, \mathrm{pn}}+I_{\mathrm{Ks}}+I_{\mathrm{pn}, \mathrm{s}}+I_{\mathrm{pn}, \mathrm{d}}+I_{\mathrm{pn}, \mathrm{a}}\right), \\
& C_{\mathrm{m}} \frac{\mathrm{d} V_{\mathrm{d}}}{\mathrm{d} t}=-\left(I_{\mathrm{L}, \mathrm{d}}+I_{\mathrm{KCa}}+I_{\mathrm{Ca}}+I_{\mathrm{A}}+I_{\mathrm{Kf}}+I_{\mathrm{d}, \mathrm{pn}}\right), \\
& C_{\mathrm{m}} \frac{\mathrm{d} V_{\mathrm{a}}}{\mathrm{d} t}=I_{\mathrm{ext}}-\left(I_{\mathrm{L}, \mathrm{a}}+I_{\mathrm{Na}}+I_{\mathrm{Kdr}}+I_{\mathrm{a}, \mathrm{pn}}\right),
\end{aligned}
$$


where $V_{\mathrm{s}}, V_{\mathrm{pn}}, \quad V_{\mathrm{d}}$ and $V_{\mathrm{a}}$ represent the membrane potential of soma, primary neurite, dendrite and axon, respectively. $I_{\text {ext }}$ is external stimulus. $C_{\mathrm{m}}$ is the membrane capacitance and equals to $1 \mathrm{nF}$. The coupling current $I_{\mathrm{i}, \mathrm{j}}$ between compartment i and compartment $\mathrm{j}$ is described by $I_{\mathrm{i}, \mathrm{j}}=G_{\mathrm{i}, \mathrm{j}}\left(V_{\mathrm{i}}-V_{\mathrm{j}}\right), G_{\mathrm{i}, \mathrm{j}}$ is the coupling strength, $G_{\mathrm{s}, \mathrm{pn}}=G_{\mathrm{pn}, \mathrm{s}}=0.05 \mu \mathrm{S}, G_{\mathrm{a}, \mathrm{pn}}=G_{\mathrm{pn}, \mathrm{a}}=0.5 \mu \mathrm{S}$, $G_{\mathrm{d}, \mathrm{pn}}=G_{\mathrm{pn}, \mathrm{d}}=0.04 \mu \mathrm{S}$. The current of each ion channel follows the equations below.

$$
\begin{aligned}
& I_{\mathrm{Na}}=g_{\mathrm{Na}} m^{3} h\left(V-E_{\mathrm{Na}}\right), \\
& m=\frac{a_{\mathrm{m}}}{a_{\mathrm{m}}+b_{\mathrm{m}}}, \frac{\mathrm{d} h}{\mathrm{~d} t}=a_{\mathrm{h}}(1-h)-b_{\mathrm{h}} h, \\
& a_{\mathrm{m}}=\frac{0.121 V+2.871}{1-\exp (-0.121 V-2.871)}, b_{\mathrm{m}}=4 \exp (-0.0672 V-2.984), \\
& a_{\mathrm{h}}=0.07 \exp (-0.0605 V-2.686), b_{\mathrm{h}}=\frac{1}{1+\exp (-0.121 V-2.371)} . \\
& I_{\mathrm{Kdr}}=g_{\mathrm{Kdr}} n^{4}\left(V-E_{\mathrm{K}}\right), \frac{\mathrm{d} n}{\mathrm{~d} t}=a_{\mathrm{n}}(1-n)-b_{\mathrm{n}} n, \\
& a_{\mathrm{n}}=\frac{0.121 V+2.237}{1-\exp (-0.121 V-2.429)}, b_{\mathrm{n}}=0.125 \exp (-0.0151 V-0.421) . \\
& I_{\mathrm{KCa}}=g_{\mathrm{KCa}} \frac{c}{0.5+c}\left(V-E_{\mathrm{K}}\right), \frac{\mathrm{d} c}{\mathrm{~d} t}=\rho\left(\frac{0.0078 z\left(E_{\mathrm{Ca}}-V\right)}{1+2 c}-c\right) . \\
& I_{\mathrm{Ca}}=g_{\mathrm{Ca}} \frac{z}{0.43+z}\left(V-E_{\mathrm{Ca}}\right) \text {, } \\
& \frac{\mathrm{d} z}{\mathrm{~d} t}=\frac{z_{\mathrm{V}}-z}{23}, z_{\mathrm{V}}=\frac{1}{1+\exp (-0.15 V-7.5)} . \\
& I_{\mathrm{A}}=g_{\mathrm{A}} m_{\mathrm{A}}^{3} h_{\mathrm{A}}\left(V-E_{\mathrm{K}}\right), m_{\mathrm{A}}=\frac{1}{1+\exp \left(-\frac{V+12}{26}\right)}, \\
& \frac{\mathrm{d} h_{\mathrm{A}}}{\mathrm{d} t}=h_{\mathrm{Ai}}-h_{\mathrm{A}}, h_{\mathrm{Ai}}=\frac{1}{1+\exp \left(\frac{V+62}{6}\right)} . \\
& I_{\mathrm{Ks}}=g_{\mathrm{Ks}} p\left(V-E_{\mathrm{K}}\right), \frac{\mathrm{d} p}{\mathrm{~d} t}=\frac{p_{\mathrm{V}}-p}{\tau_{\mathrm{p}}}, \\
& p_{\mathrm{V}}=\frac{1}{1+\exp (-2 V-90)}, \tau_{\mathrm{p}}=100+\frac{3000}{1+\exp \left(-\frac{V+50}{0.05}\right)} . \\
& I_{\mathrm{Kf}}=g_{\mathrm{Kf}} b\left(V-E_{\mathrm{K}}\right) \text {, } \\
& \frac{\mathrm{d} b}{\mathrm{~d} t}=b_{\mathrm{V}}-b, b_{\mathrm{V}}=\frac{1}{1+\exp (-2 V-84)} .
\end{aligned}
$$




\section{Neural Network World 2/2017, 247-258}

$$
I_{\mathrm{L}, \mathrm{i}}=g_{\mathrm{L}, \mathrm{i}}\left(V-E_{\mathrm{L}}\right) .
$$

The value of conductances in the equations above are $g_{\mathrm{Na}}=15 \mu \mathrm{S}, \quad g_{\mathrm{Kdr}}=$ $8 \mu \mathrm{S}, \quad g_{\mathrm{KCa}}=0.273 \mu \mathrm{S}, g_{\mathrm{Ca}}=0.04 \mu \mathrm{S}, \quad g_{\mathrm{A}}=100 \mu \mathrm{S}, \quad g_{\mathrm{L}, \mathrm{s}}=g_{\mathrm{L}, \mathrm{a}}=g_{\mathrm{L}, \mathrm{pn}}=$ $0.001 \mu \mathrm{S}, g_{\mathrm{L}, \mathrm{d}}=0.0354 \mu \mathrm{S}, g_{\mathrm{Ks}}=0.065 \mu \mathrm{S}, g_{\mathrm{Kf}}=0.07 \mu \mathrm{S}$. And the corresponding equilibrium potentials are $E_{\mathrm{Na}}=30 \mathrm{mV}, E_{\mathrm{K}}=-75 \mathrm{mV}, E_{\mathrm{Ca}}=140 \mathrm{mV}, E_{\mathrm{L}}=$ $-40 \mathrm{mV}$. 